\title{
Usage of Cholesky Decomposition in order to Decrease the Nonlinear Complexities of Some Nonlinear and Diversification Models and Present a Model in Framework of Mean-Semivariance for Portfolio Performance Evaluation
}

\author{
H. Siaby-Serajehlo, ${ }^{1}$ M. Rostamy-Malkhalifeh, ${ }^{1}$ F. Hosseinzadeh Lotfi, ${ }^{1}$ and M. H. Behzadi ${ }^{2}$ \\ ${ }^{1}$ Department of Mathematics, Science and Research Branch, Islamic Azad University, Tehran, Iran \\ ${ }^{2}$ Department of Statistics, Science and Research Branch, Islamic Azad University, Tehran, Iran
}

Correspondence should be addressed to M. Rostamy-Malkhalifeh; mohsen_rostamy@yahoo.com

Received 22 October 2015; Revised 12 February 2016; Accepted 14 February 2016

Academic Editor: Shey-Huei Sheu

Copyright (C) 2016 H. Siaby-Serajehlo et al. This is an open access article distributed under the Creative Commons Attribution License, which permits unrestricted use, distribution, and reproduction in any medium, provided the original work is properly cited.

\begin{abstract}
In order to get efficiency frontier and performance evaluation of portfolio, nonlinear models and DEA nonlinear (diversification) models are mostly used. One of the most fundamental problems of usage of nonlinear and diversification models is their computational complexity. Therefore, in this paper, a method is presented in order to decrease nonlinear complexities and simplify calculations of nonlinear and diversification models used from variance and covariance matrix. For this purpose, we use a linear transformation which is obtained from the Cholesky decomposition of covariance matrix and eliminate linear correlation among financial assets. In the following, variance is an appropriate criterion for the risk when distribution of stock returns is to be normal and symmetric as such a thing does not occur in reality. On the other hand, investors of the financial markets do not have an equal reaction to positive and negative exchanges of the stocks and show more desirability towards the positive exchanges and higher sensitivity to the negative exchanges. Therefore, we present a diversification model in the mean-semivariance framework which is based on the desirability or sensitivity of investor to positive and negative exchanges, and rate of this desirability or sensitivity can be controlled by use of a coefficient.
\end{abstract}

\section{Introduction}

Despite the thousands of investment mutual funds for the rendering services to the investors and high volume of financial transactions accomplished in the these mutual funds [1] and with regard to the plentiful studies performed in this field, no discussions can be raised that portfolio performance evaluation and investment mutual funds' performance evaluation play an effective role in the decision making of the investors in order to invest in the financial markets. Various investors use different definitions of performance evaluation depending on their perspective and outlook in order to predict financial markets.

One of the most important viewpoints in the portfolio performance evaluation is to use portfolio efficiency frontier. In this method, distance of the desired portfolio from efficiency frontier is the considered a criterion for portfolio performance evaluation. Markowitz [2] presented a model in the mean-variance framework for portfolio performance evaluation using this criterion. In this model, existence of the linear correlation among financial assets has led to existence of nonlinear complexities in the model. During recent years, the researches carried out in this field have been concentrated on the results of this theory. Sharpe [3] presented a model in order to decrease the volume of covariance matrix calculations (linear correlation among the financial assets) using return market index. In this method, linear correlation among the financial assets is considered to be zero. Instead, using regression, return of each financial assets is related to return market index which is referred to as single-index model. Usage of Markowitz model involves existence of enough data regarding mean, variance, and 
covariance between each two pairs of financial assets which include $(n(n+3) / 2)$ of data. Now, if the sharpe singleindex model is used, required data will decrease to ( $3 n-$ 2 ). On the other hand, in the single-index model, it was assumed that return of one asset is only affected by an index (market index) but, due to available weaknesses and lack of ignoring of all indexes affecting the performance evaluation, some researchers created multi-index models via addition of the indexes and nonmarket factors (inflation rate, foreign exchange rate, oil price, industry index, etc.) [4]. M. R. Morey and R. C. Morey presented a diversification model inspired by DEA [5]. Also, in this model, existence of linear correlation among financial assets has been led to existence of the nonlinear complexities in the model. Briec et al. [6] presented a diversification model in the mean-variance framework through introduction of efficiency improvement possibility function (shortage function). For the sake of investors' inclination towards the positive skewness [7-9], Joro and $\mathrm{Na}$ [10] offered a model in the mean-variance-skewness framework by consideration of the variance as input and skewness and mean as output. In some studies, semivariance has been used instead of variance because variance is counted as an appropriate criterion for the risk when the stocks return distribution is to be in normal and symmetric form, which it is not in such a manner most of times [11, 12].

One of our basic problems is complexity of the calculations of diversification and nonlinear models. We usually use Lagrange method, KKT method [13], and various heuristic methods [14] in order to solve these models in case of having the required conditions. For example, in the Lagrange or KKT method, we solve the problem by transformation of optimization problem into linear systems. In order to solve linear systems, there are various methods which, for example, can be referred to the direct methods such as Gaussian and Gaussian-Jordan elimination methods and matrix decomposition methods such as Lu decomposition, orthogonal matrixes, and Cholesky decomposition [15]. Cholesky decomposition can be applied for the matrixes which are positive definite and symmetric. Solving linear systems is one of the principal applications of the Cholesky decomposition. Another one of its application is data production for dependent variable using simulation [16, 17]. In this paper, we, at first, present a linear transformation based on Cholesky decomposition. In continuation, we present a diversification model in the mean-semivariance framework and, using the mentioned linear transformation, decrease nonlinear computational complexities of the Markowitz, M. R. Morey and R. C. Morey, Briec et al., and suggestive models.

This paper has been classified as follows: in Section 2, we present a brief description in connection with the Cholesky decomposition, positive definite matrix, covariance matrix, and their properties and finish the section through introduction of a linear transformation and financial interpretation of the Cholesky decomposition. In Section 3, we apply the mentioned linear transformation for Markowitz, M. R. Morey, R. C. Morey, and Briec et al. models and express advantages of the obtained models. In Section 4, we present a model in the mean-semivariance framework and apply the mentioned linear transformation for that and, at the end, the potential integration of higher moments, briefly is discussed. Section 5 includes numerical examples for explanation of the methods and suggestive models. We finish the paper with conclusion in Section 6.

\section{Required Concepts}

In this section, we take a look at the required concepts briefly and deal with their properties by presentation of a linear transformation.

Definition 1. $A_{n \times n}$ (square matrix) is known as positive definite matrix whenever

$$
\forall x\left(x \in \mathbb{R}^{n}, x \neq 0\right) \Longrightarrow x^{T} A x>0 .
$$

Definition 2 (Cholesky decomposition). If $A_{n \times n}$ is symmetric and positive definite matrix then there exists a unique lower triangular matrix $L_{n \times n}$ with positive diagonal element such that

$$
A=L L^{T}
$$

Consider the vector random variable $X=\left\{x_{1}, \ldots, x_{n}\right\}$ which has $E(X)$ (mean vector) and $\operatorname{var}(X)$ (covariance matrix). $\operatorname{var}(X)$ is a positive and symmetrical definite matrix. Therefore, we will use Cholesky decomposition as follows:

$$
\begin{aligned}
\operatorname{var}(X) & =\left[\operatorname{cov}\left(x_{i}, x_{j}\right)\right]_{n \times n}=L L^{T}, \\
L & =\left[\begin{array}{cccc}
L_{11} & 0 & \cdots & 0 \\
L_{21} & L_{22} & \cdots & 0 \\
\vdots & \vdots & & \vdots \\
L_{n 1} & L_{n 2} & \cdots & L_{n n}
\end{array}\right]
\end{aligned}
$$

Consider the linear transformation presented in the following relation:

$$
W=L^{-1} X
$$

We prove that the linear correlation between the random variables of $W$ is equal to zero:

$$
\begin{aligned}
L L^{T} & =\operatorname{var}(X)=\operatorname{var}(L W) \\
& =E\left[(L W-L E(W))(L W-L E(W))^{T}\right] \\
& =L \operatorname{var}(W) L^{T} .
\end{aligned}
$$

Therefore

$$
\begin{aligned}
\operatorname{var}(W) & =I \Longrightarrow \\
\operatorname{cov}\left(w_{i}, w_{j}\right)_{i \neq j} & =0 .
\end{aligned}
$$

2.1. Financial Interpretation of the Cholesky Decomposition. Cholesky decomposition technique is one of the available methods for data production from multivariate distributions, 
because we require data production and simulation in some problems due to historical insufficiencies, lack of enough knowledge concerning future process of variables, and so forth, use statistical properties of historical data, and present random variable $X$ with a specified distribution. Now, if this random variable $X$ is independent, we will deal with data production using simulation easily, but if we confront with a few variables or a series of random observations having dependence, action cannot be taken any more to simulate each one of the random variables without consideration of their dependence on other variables. In order to solve this problem, we use the following three-step process:

(1) Transfer of data to a space in which there is not any dependence among the variables.

(2) Production and simulation of data in this new space.

(3) Transfer of produced data to the initial space.

One of the main methods to perform this job is Cholesky decomposition.

For other cases of Cholesky decomposition usage, refer to the multivariate options evaluation [18]. Cholesky decomposition plays an important role in analysis of the risk factors, putting price on financial markets and risk management; for example, refer to the VAR models [19] and copula models [20].

With regard to Cholesky decomposition properties, we transfer financial assets from initial space to a new space considering financial assets as random variables and using the linear transformation presented in this paper. In the new space, linear correlation among financial assets becomes zero and standard deviation of financial assets becomes equal to one. Therefore, nonlinear complexities of the mentioned models are decreased in the new spaces.

\section{Reduction of Nonlinear Complexities of the Markowitz, M. R. Morey, R. C. Morey, and Briec et al. Models}

In this section, we implement linear transformation of relation (4) on the Markowitz, M. R. Morey, and R. C. Morey models and compare the models obtained from linear transformation with principal models. Before beginning the discussion, we get the relations using the mentioned linear transformation and use them until the end of the paper. Let us suppose that we possess $n$ financial assets or $n$ portfolios like random variable $x_{i}$. We consider the following assumptions:

$$
\begin{aligned}
E\left(x_{i}\right) & =\bar{\mu}_{i}, \\
E\left(\left(x_{i}-\bar{\mu}_{i}\right)^{2}\right) & =\bar{\sigma}_{i}^{2}, \\
\operatorname{cov}\left(x_{i}, x_{j}\right) & =\bar{\sigma}_{i j}, \\
E\left(w_{i}\right) & =\mu_{i}, \\
E\left(\left(w_{i}-\mu_{i}\right)^{2}\right) & =\sigma_{i}^{2}, \\
\operatorname{cov}\left(w_{i}, w_{j}\right) & =\sigma_{i j} .
\end{aligned}
$$

Now, using linear transformation of $W=L^{-1} X$ and relation of $B=L^{-1}$, we have the following:

$$
\sum_{j=1}^{n} \lambda_{j} x_{j}=\sum_{j=1}^{n} \alpha_{j} w_{j},
$$

where $\alpha_{j}$ is defined as follows:

$$
\left[\lambda_{1}, \ldots, \lambda_{n}\right] L=\left[\alpha_{1}, \ldots, \alpha_{n}\right] .
$$

Of relation (9), we will have

$$
\left[\lambda_{1}, \ldots, \lambda_{n}\right]=\left[\alpha_{1}, \ldots, \alpha_{n}\right] B
$$

The following relation can be concluded from relation (10):

$$
\begin{aligned}
\sum_{j=1}^{n} \lambda_{j} & =1 \Longrightarrow \\
\sum_{j=1}^{n} B_{j} \alpha_{j} & =1,
\end{aligned}
$$

where $B_{j}$ is sum total entries of the $j$ th row in matrix $L^{-1}$. In continuation, using relation (8), we have

$$
\begin{array}{r}
E\left(x_{p}\right)=E\left(\sum_{j=1}^{p} L_{p j} w_{j}\right)=\sum_{j=1}^{p} L_{p j} \mu_{j}, \\
E\left(\sum_{j=1}^{n} \lambda_{j} x_{j}\right)=E\left(\sum_{j=1}^{n} \alpha_{j} w_{j}\right)=\sum_{j=1}^{n} \alpha_{j} \mu_{j} .
\end{array}
$$

Using relations (6), (7), (8), and (12), the following relations are achieved:

$$
\begin{aligned}
& E\left(\left(x_{p}-\bar{\mu}_{p}\right)^{2}\right)=\sum_{j=1}^{p}\left(L_{p j}\right)^{2}, \\
& E\left(\left(\sum_{j=1}^{n} \lambda_{j}\left(x_{i}-\bar{\mu}_{i}\right)\right)^{2}\right) \\
& \quad=E\left(\left(\sum_{j=1}^{n} \alpha_{j}\left(w_{j}-\mu_{j}\right)\right)^{2}\right)=\sum_{j=1}^{n} \alpha_{j}^{2} .
\end{aligned}
$$

3.1. Markowitz Model. In the Markowitz model, we consider the mean to be constant in the specified levels and make the variance to be minimum in order to obtain portfolio efficiency frontier:

$$
\begin{array}{ll}
\min & \sum_{j=1}^{n} \lambda_{j}^{2} \bar{\sigma}_{j}^{2}+\left(\sum_{i=1}^{n} \sum_{j=1}^{n} \lambda_{i} \lambda_{j} \bar{\sigma}_{i j}\right)_{i \neq j} \\
\text { s.t. } & \sum_{j=1}^{n} \lambda_{j} \bar{\mu}_{j}=R_{\text {expected }}, \\
& \sum_{j=1}^{n} \lambda_{j}=1, \\
& \lambda_{j} \geq 0, j=1, \ldots, n .
\end{array}
$$


In continuation, through implementation of the linear transformation (4) and placement of relations (6), (10), (11), (13), and (15) in the model (16), we reach the following model:

$$
\begin{array}{ll}
\min & \sum_{j=1}^{n} \alpha_{j}^{2} \\
\text { s.t. } & \sum_{j=1}^{n} \alpha_{j} \mu_{j}=R_{\text {expected }}, \\
& \sum_{j=1}^{n} B_{j} \alpha_{j}=1, \\
& \sum_{j=1}^{n} B_{j i} \alpha_{j} \geq 0, \quad i=1, \ldots, n,
\end{array}
$$

where $B_{j i}$ is the same as $L_{j i}^{-1}$. It is evident that models (16) and (17) are equivalent. We consider objective function of model (16) which has $\left(n^{2}+n\right) / 2$ nonlinear combinations of variables in form of $\lambda_{j}^{2}$ and $\lambda_{i} \lambda_{j}$, but in the objective function of model (17) nonlinear combinations of variables decrease up to $n$ which is in the form of $\alpha_{j}^{2}$. In fact, through implementation of the introduced linear transformation, we decrease the nonlinear complexities of model (16) and point out that, while solving model (17) by use of common methods of solving nonlinear models, calculations will be less and easier. With regard to solving the majority of the nonlinear problems using heuristic methods (the obtained solution is approximate), we can solve the problem quickly and accurately using model (17) while facing a great volume of data in a portfolio optimization problem. It is required to mention that, while solving model (16) and model (17) with KKT method, size of Hessian matrix in model (16) is equal to $n^{2}$, and size of Hessian matrix in model (17) is equal to $n$.

3.2. M. R. Morey and R. C. Morey Model. M. R. Morey and R. C. Morey used the following model in order to evaluate portfolio performance. In this model, they consider the mean as output and variance as input:

$$
\begin{array}{ll}
\min & \theta \\
\text { s.t. } & \sum_{j=1}^{n} \lambda_{j} \bar{\mu}_{j} \geq \bar{\mu}_{p}, \\
& \sum_{j=1}^{n} \lambda_{j}^{2} \bar{\sigma}_{j}^{2}+\left(\sum_{i=1}^{n} \sum_{j=1}^{n} \lambda_{i} \lambda_{j} \bar{\sigma}_{i j}\right)_{i \neq j} \leq \theta \bar{\sigma}_{p}^{2}, \\
& \sum_{j=1}^{n} \lambda_{j}=1, \\
& \lambda_{j} \geq 0, \quad j=1, \ldots, n .
\end{array}
$$

By implementation of the linear transformation (4) and placement of relations (6), (10), (11), (12), (13), (14), and (15) in the model (18), we get the following model:

$$
\begin{array}{ll}
\min & \theta \\
\text { s.t. } & \sum_{j=1}^{n} \alpha_{j} \mu_{j} \geq \sum_{j=1}^{p} L_{p j} \mu_{j}, \\
& \sum_{j=1}^{n} \alpha_{j}^{2} \leq \theta \sum_{j=1}^{p}\left(L_{p j}\right)^{2}, \\
& \sum_{j=1}^{n} B_{j} \alpha_{j}=1, \\
& \sum_{j=1}^{n} B_{j i} \alpha_{j} \geq 0, \quad i=1, \ldots, n .
\end{array}
$$

It is evident that models (18) and (19) are equivalent. The second constraint of model $(18)$ has $\left(n^{2}+n\right) / 2$ nonlinear combinations of variables in form of $\lambda_{j}^{2}$ and $\lambda_{i} \lambda_{j}$, but, in the second constraint of model (19) nonlinear combinations of variables decrease to $n$ which is in form of $\alpha_{j}^{2}$. Also, here, through implementation of the introduced linear transformation, the nonlinear complexities of model (18) are decreased, and the examples with high data number can be worked on more easily due to simplicity of the calculations. Also, size of Hessian matrix in model (18) and model (19) is equal to $n^{2}$ and $n$, respectively.

3.3. Briec et al. Model. For portfolio performance evaluation, Briec et al. [6] used the following model. In this model, they considered mean as output and variance as input. Efficiency improvement possibility function (shortage function) in this model searches for improvement in direction of vector $g(g=$ $\left.\left(g_{V}, g_{E}\right) \geq 0\right)$ and, simultaneously, seeks for increase of the mean and decrease of variance:

$$
\begin{array}{ll}
\max & \theta \\
\text { s.t. } & \sum_{j=1}^{n} \lambda_{j} \bar{\mu}_{j} \geq \bar{\mu}_{p}+\theta g_{E}, \\
& \sum_{j=1}^{n} \lambda_{j}^{2} \bar{\sigma}_{j}^{2}+\left(\sum_{i=1}^{n} \sum_{j=1}^{n} \lambda_{i} \lambda_{j} \bar{\sigma}_{i j}\right)_{i \neq j} \leq \bar{\sigma}_{p}^{2}-\theta g_{V}, \\
& \sum_{j=1}^{n} \lambda_{j}=1, \\
& \lambda_{j} \geq 0, j=1, \ldots, n .
\end{array}
$$


By implementation of the linear transformation (4) and placement of relations (6), (10), (11), (12), (13), (14), and (15) in model (20), we get the following model:

$$
\begin{array}{ll}
\max & \theta \\
\text { s.t. } & \sum_{j=1}^{n} \alpha_{j} \mu_{j} \geq \sum_{j=1}^{p} L_{p j} \mu_{j}+\theta g_{E}, \\
& \sum_{j=1}^{n} \alpha_{j}^{2} \leq \sum_{j=1}^{p}\left(L_{p j}\right)^{2}-\theta g_{V}, \\
& \sum_{j=1}^{n} B_{j} \alpha_{j}=1, \\
& \sum_{j=1}^{n} B_{j i} \alpha_{j} \geq 0, \quad i=1, \ldots, n .
\end{array}
$$

It is obvious that models (20) and (21) are equivalent. The second constraint of model $(20)$ has $\left(n^{2}+n\right) / 2$ nonlinear combinations of variables in form of $\lambda_{j}^{2}$ and $\lambda_{i} \lambda_{j}$, but, in the second constraint of model (21), nonlinear combinations of variables decrease up to $n$ which is in form of $\alpha_{j}^{2}$. Also, here, through implementation of the introduced linear transformation, the nonlinear complexities of model (20) are decreased. Also, size of Hessian matrix in model (20) and model (21) is equal to $n^{2}$ and $n$, respectively.

\section{Presentation of the Diversification Model in the Mean-Semivariance Framework}

Consideration of variance criterion instead of risk criterion is counted as an appropriate criterion in case distribution of random variable $X$ that is in symmetric and normal form, but such a thing does not occur in reality. Investors of financial markets do not react to the positive and negative exchanges of the stocks equally and show more sensitivity to the negative exchanges and always intend to increase positive exchanges and decrease negative exchanges. For this purpose, we present the following model in the mean-semivariance framework. We define favorable variance and unfavorable variance, respectively, as follows:

$$
\begin{aligned}
& \sigma_{\text {up }}^{2}=E\left(\left(\sum_{j \in A} \lambda_{j}\left(x_{i}-\bar{\mu}_{i}\right)\right)^{2}\right), \\
& A=\left\{j \mid x_{i}-\bar{\mu}_{i} \geq 0, j=1, \ldots, n\right\}, \\
& \sigma_{\text {down }}^{2}=E\left(\left(\sum_{j \in B} \lambda_{j}\left(x_{i}-\bar{\mu}_{i}\right)\right)^{2}\right), \\
& B=\left\{j \mid x_{i}-\bar{\mu}_{i}<0, j=1, \ldots, n\right\} .
\end{aligned}
$$

We present the following diversification model in the mean-semivariance framework which we consider as output and combination of the favorable and unfavorable variance as input:

$$
\begin{array}{ll}
\min & \theta \\
\text { s.t. } & \sum_{j=1}^{n} \lambda_{j} \bar{\mu}_{j} \geq \bar{\mu}_{p}, \\
& E\left(\left(\sum_{j=1}^{n} \beta y_{j} \lambda_{j}\left(x_{j}-\bar{\mu}_{j}\right)+\sum_{j=1}^{n}(1-\beta)\left(1-y_{j}\right) \lambda_{j}\left(x_{j}-\bar{\mu}_{j}\right)\right)\right) \\
& \leq \theta E\left(\left(\beta y_{p}\left(x_{p}-\bar{\mu}_{p}\right)+(1-\beta)\left(1-y_{p}\right)\left(x_{p}-\bar{\mu}_{p}\right)\right)^{2}\right), \\
& \sum_{j=1}^{n} \lambda_{j}=1, \\
& y_{j}\left(x_{j}-\bar{\mu}_{j}\right) \geq 0, \quad j=1, \ldots, n, \\
& \left(1-y_{j}\right)\left(x_{j}-\bar{\mu}_{j}\right) \leq 0, \quad j=1, \ldots, n, \\
& \lambda_{j} \geq 0, j=1, \ldots, n, \\
& y_{j} \in\{0,1\}, j=1, \ldots, n .
\end{array}
$$

Scalar of $0 \leq \beta \leq 1$ is specified by the investor. As much as $\beta$ gets close to one, it suggests more priority of the positive exchanges over negative exchanges (with an optimistic vision, investor deals with portfolio performance evaluation), and 
anywhere it gets close to zero, it suggests higher sensitivity of the investor to negative exchanges (with a pessimistic vision, investor deals with portfolio performance evaluation). Model (23) is general state of model (18) (it is sufficient to place $\beta=0.5)$. A portfolio has a better performance which possesses a higher $\theta$. Excellence of the presented model is consideration of the linear correlation among the financial assets because linear correlation among financial assets has been considered to be zero in most of models which have used semivariance. Now, using linear transformation (4) and implementing relations (6), (10), (11), (12), (13), (14), and (15) in the model (23), the following model can be presented:

$$
\begin{array}{ll}
\min & \theta \\
\text { s.t. } \quad & (1-\beta)^{2} \sum_{j=1}^{n} \alpha_{j}^{2}+(2 \beta-1) \sum_{j=1}^{n} y_{j} \alpha_{j}^{2} \\
& \leq \theta \sum_{j=1}^{p}\left(L_{p j}\right)^{2}\left((1-\beta)^{2}+(2 \beta-1) y_{p}\right), \\
& \sum_{j=1}^{n} B_{j} \alpha_{j}=1, \\
& \sum_{j=1}^{n} B_{j i} \alpha_{j} \geq 0, \quad i=1, \ldots, n, \\
& \left(\sum_{i=1}^{j} L_{j i}\left(w_{i}-\mu_{i}\right) y_{j}\right) \geq 0, \quad j=1, \ldots, n, \\
& \left(\sum_{i=1}^{j} L_{j i}\left(w_{i}-\mu_{i}\right)\right)\left(1-y_{j}\right) \leq 0, \\
& y_{j} \in\{0,1\}, j=1, \ldots, n .
\end{array}
$$

It is evident that models (23) and (24) are equivalent. The second constraint of model $(23)$ has $\left(n^{2}+n\right) / 2$ nonlinear combinations of variables in form of $y_{j}^{2} \lambda_{j}^{2}$ and $y_{i} y_{j} \lambda_{i} \lambda_{j}$, but, in the second constraint of model (24), nonlinear combinations of variables have been reduced to $n$, appearing in form of $y_{j} \alpha_{j}^{2}$. Also, here, model (24) has less nonlinear complexities than model (23) and the examples with high data number can be worked on easily due to simplicity of the calculations.

At the end, we are concerned with reviewing a summary of higher moments. As it was stated, timing meanvariance framework is construed as an appropriate criterion for portfolio performance evaluation, in which distribution of random variables $X$ (financial assets and portfolio) is in symmetrical and normal form. But Mandelbrot [21] showed that such a thing does not occur in reality. In order to analyze these problems, Popova et al. [22] and Davies et al. [23] used higher moments; for example, refer to skewness and kurtosis [24] as follows:

$$
\begin{aligned}
E(R) & =E\left(\sum_{j=1}^{n} \lambda_{j} x_{j}\right)=\sum_{j=1}^{n} \lambda_{j} \mu_{j}, \\
\delta^{2} & =E\left[(R-E(R))^{2}\right]=\sum_{i=1}^{n} \sum_{j=1}^{n} \lambda_{i} \lambda_{j} \sigma_{i j}, \\
S^{3} & =E\left\{(R-E(R))^{3}\right\}=\sum_{i=1}^{n} \sum_{j=1}^{n} \sum_{k=1}^{n} \lambda_{i} \lambda_{j} \lambda_{k} S_{i j k}, \\
\mathcal{K}^{4} & =E\left\{(R-E(R))^{4}\right\} \\
& =\sum_{i=1}^{n} \sum_{j=1}^{n} \sum_{k=1}^{n} \sum_{l=1}^{n} \lambda_{i} \lambda_{j} \lambda_{k} \lambda_{l} \kappa_{i j k l} .
\end{aligned}
$$

Relations (25) and (26) are the same as mean return and variance and considered to be the main framework discussed by this paper. Relation (27) suggests skewness or the third central moment. In fact, rate of deviation from symmetry of a distribution is known as skewness. Of the models presented in the mean-variance skewness (MVS) framework as an example, it can be referred to [10, 25]. We were reminded earlier that investors are interested highly in the positive skewness and consider the skewness as the output in the diversification models. Relation (28) represents kurtosis or the fourth central moment. Kurtosis shows the same amount of climax or higher distribution compared to normal distribution. Of the models presented in the mean-variance-skewness-kurtosis (MVSK) frame work as an example, it can be referred to [26-28]. Considering the conducted researches, investors are interested in low kurtosis and, therefore, consider the kurtosis is input in the diversification models. At the end, we refer to this subject that linear transformation presented in this paper can be used to decrease nonlinear complexities of the nonlinear and diversification models in MVS and MVSK frameworks, but, perhaps, this decrease is not tangible so much, yet this decrease is tangible in MV framework completely.

\section{Numerical Example}

In this section, we deal with presentation of three numerical examples in order to describe the suggestive methods and models. We point out that the examples have been solved by use of GAMS (24.1.2) Software, MATLAB (2012) Software and Windows (8.1) Oprating System.

Example 1. Consider the data related to 26 financial assets with mean and covariance matrix presented in Table 3 [5]. In this example, we are going to obtain portfolios efficiency frontier achieved from the mentioned financial assets using model (16) and model (17). For this purpose, it is sufficient to solve model (16) and model (17) for a number of the fixed mean levels. The results can be seen in Table 1.

With regard to equality (analytical) of models (16) and (17), the obtained results for both models must be equal, but, 
TABLE 1: Solution of models (16) and (17) for a number of the fixed mean levels.

\begin{tabular}{|c|c|c|c|c|c|c|c|c|c|c|c|c|c|c|c|}
\hline$R_{\text {expected }}$ & 1.737 & 1.074 & 1.543 & 1.791 & 1.033 & 1.463 & 1.368 & 1.367 & 0.985 & 1.165 & 1.303 & 1.349 & 1.411 & 1.114 & 1.385 \\
\hline Model (16) & 40.314 & 20.048 & 50.070 & 45.58 & 67 & 28. & 25. & 29 & 18.99 & 38 & 22 & 1 & 44 & 69 & 26 \\
\hline Model (17) & 40.299 & 20.040 & 30.664 & 45.562 & 19.459 & 28.331 & 25.842 & 25.817 & 18.987 & 21.528 & 24.311 & 25.380 & 26.931 & 20.660 & 26.265 \\
\hline
\end{tabular}

in reality, answers of these two models are equal up to one decimal digit, and answers of model (17) are less than or equal to answers of the model (16). As a result, due to minimization of the problem, it can be concluded that answers of model (17) have a better numerical approximation. In fact, when we use KKT method in order to solve models (16) and (17), size of Hessian matrix used for model (16) will be equal to 676 and for model (17) will be equal to 26. Therefore, GAMS software solves model (17) within a less time span compared to model (16). Now, if we have 1000 financial assets instead of 26 financial assets, this time difference will be remarkable.

Example 2. Let us suppose that we consider the data related to 26 financial assets, with mean and covariance matrix given in Table 3, as portfolios. In this example, we deal with evaluation of the mentioned portfolios performance. Considering equality (analytical) of models (18) and (19), the achieved results for both models must be equal, but, in reality, answers of these two models are equal up to two decimal digits, and answers of model (19) are less than or equal to answers of model (18) (Table 2). Therefore, due to minimization of the problem, it can be concluded that answer of model (19) has a better numerical approximation. In fact, when we use KKT method in order to solve models (18) and (19), size of Hessian matrix used for model (18) will be equal to 676 and for model (19) will be equal to 26. Thus, GAMS software solves model (19) within a less time span compared to model (18).

The results of Example 2 can be considered for models (20) and (21). Therefore, due to similar results, we avoid to provide any additional example.

Example 3. Consider the data related to 26 portfolios of Example 2. In this example, we want to evaluate the portfolios performance using model (24). For simplicity, let us suppose that odd portfolios have favorable semivariance and even portfolios have unfavorable semivariance. Therefore

$$
\begin{aligned}
& y_{1}=y_{3}=\cdots=y_{25}=1, \\
& y_{2}=y_{4}=\cdots=y_{26}=0 .
\end{aligned}
$$

We solve model (24) for various $\beta$ (Table 2). If we consider $\beta=0.5$, portfolios number 4 and number 9 will have the best performance. With an impartial vision, performance of these two portfolios is close to each other and they have no priority over one another, but if we consider $\beta=0.4$ (Pessimistic vision), portfolio number 9 will have the best performance, and if we consider $\beta=0.6$ (Optimistic vision), portfolio number 4 will have the best performance. So, the selective portfolio changes via change of $\beta$. On the other hand, GAMS software solves model (24) due to the same reasons stated in Example 2 within a less time span compared to model (23).

\begin{tabular}{|c|c|c|c|c|c|}
\hline \multirow{2}{*}{ Portfolio } & \multirow{2}{*}{ Model (18) } & \multirow{2}{*}{ Model (19) } & \multicolumn{3}{|c|}{ Model (21) } \\
\hline & & & $\beta=0.4$ & $\beta=0.5$ & $\beta=0.6$ \\
\hline 1 & 0.7423 & 0.7422 & 0.7946 & 0.7422 & 0.7189 \\
\hline 2 & 0.3552 & 0.3551 & 0.1568 & 0.3251 & 0.7007 \\
\hline 3 & 0.6933 & 0.6932 & 0.733 & 0.6932 & 0.6744 \\
\hline 4 & 1 & 0.9996 & 0.9027 & 0.9996 & 2.1554 \\
\hline 5 & 0.5055 & 0.5054 & 0.551 & 0.5054 & 0.4827 \\
\hline 6 & 0.6201 & 0.62 & 0.292 & 0.62 & 1.3565 \\
\hline 7 & 0.6062 & 0.6061 & 0.6448 & 0.6061 & 0.5886 \\
\hline 8 & 0.8138 & 0.8136 & 0.3847 & 0.8136 & 1.7778 \\
\hline 9 & 1 & 0.9998 & 1.093 & 0.9998 & 0.9584 \\
\hline 10 & 0.7211 & 0.7209 & 0.3451 & 0.7209 & 1.5633 \\
\hline 11 & 0.6462 & 0.6461 & 0.6895 & 0.6461 & 0.6264 \\
\hline 12 & 0.8188 & 0.8187 & 0.3874 & 0.8187 & 1.7881 \\
\hline 13 & 0.6213 & 0.6212 & 0.6596 & 0.6212 & 0.6037 \\
\hline 14 & 0.6976 & 0.6974 & 0.3552 & 0.6974 & 1.5076 \\
\hline 15 & 0.645 & 0.6449 & 0.6855 & 0.6449 & 0.6265 \\
\hline 16 & 0.9803 & 0.9803 & 0.4655 & 0.9803 & 2.1241 \\
\hline 17 & 0.9718 & 0.9716 & 1.0283 & 0.9716 & 0.9451 \\
\hline 18 & 0.4413 & 0.4412 & 0.2144 & 0.4412 & 0.9516 \\
\hline 19 & 0.6857 & 0.6855 & 0.7394 & 0.6855 & 0.6599 \\
\hline 20 & 0.6387 & 0.6387 & 0.3007 & 0.6387 & 1.3976 \\
\hline 21 & 0.8097 & 0.8097 & 0.8631 & 0.8097 & 0.7806 \\
\hline 22 & 0.566 & 0.5659 & 0.2708 & 0.5659 & 1.2275 \\
\hline 23 & 0.4953 & 0.4952 & 0.5414 & 0.4952 & 0.4747 \\
\hline 24 & 0.8223 & 0.8221 & 0.3921 & 0.8221 & 1.7874 \\
\hline 25 & 0.524 & 0.5236 & 0.5724 & 0.5236 & 0.5019 \\
\hline 26 & 0.8581 & 0.8579 & 0.4113 & 0.8579 & 1.8582 \\
\hline
\end{tabular}

TABle 2: Obtained efficiency for models (18), (19), and (24).

\section{Conclusion}

In this paper, we, at first, consider $n$ random variables ( $n$ financial assets) which have linear correlation. Then, using Cholesky decomposition of the covariance matrix, we introduced a linear transformation and converted the random variables $X$ into random variables $W$ by use of this linear transformation, in which the random variables $W$ have zero linear correlation in the new space. Later, we implemented this change of variable in the Markowitz, M. R. Morey, R. C. Morey, and Briec et al. models and decreased the nonlinear complexities of this model. In continuation, since distribution of random variable $X$ is not normal and symmetric mostly, we present a new model inspired by $\mathrm{M}$. R. Morey and R. C. Morey model, using favorable and unfavorable semivariance, which is more general state of M. R. Morey and R. C. Morey model as well. The mentioned model includes a coefficient like $\beta$ showing favorability or sensitivity 


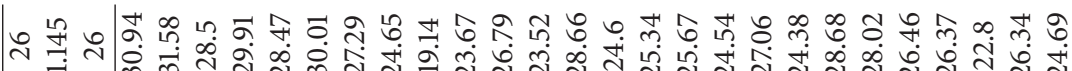

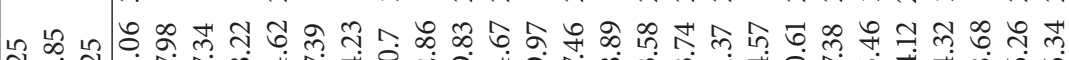

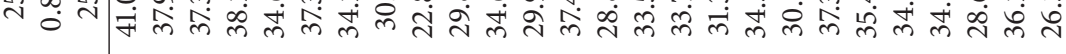
オ సै

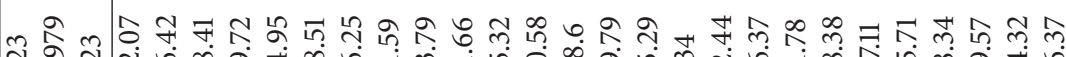

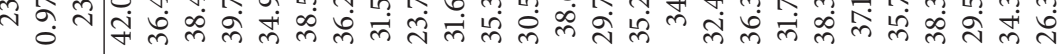

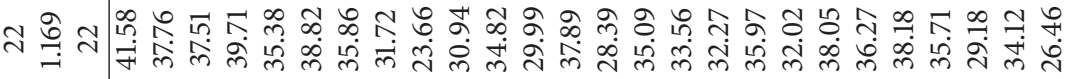

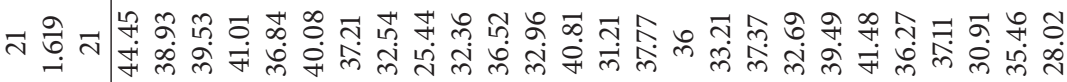

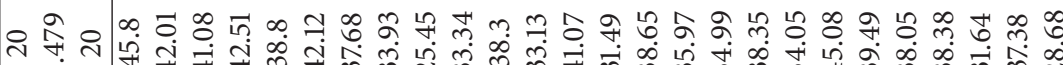

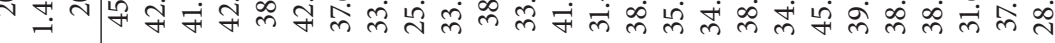

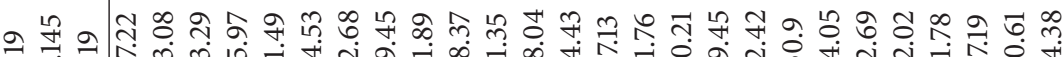

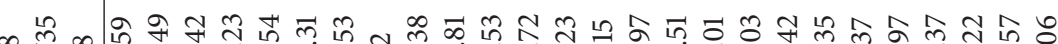

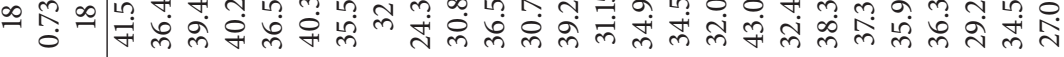

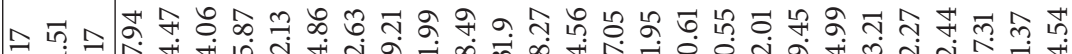

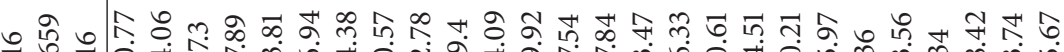

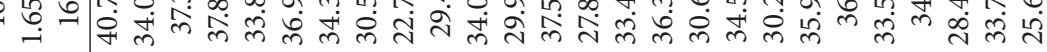
ص几 
of investor towards favorable and unfavorable semivariance. In continuation, using change of the mentioned variable, we decreased nonlinear complexities of the suggestive model.

\section{Conflict of Interests}

The authors declare that there is no conflict of interests regarding the publication of this paper.

\section{References}

[1] Time Magazine, vol. 148, no. 16, 1996.

[2] H. Markowitz, "Portfolio selection," The Journal of Finance, vol. 7, no. 1, pp. 77-91, 1952.

[3] W. F. Sharpe, "Capital asset prices: a theory of market equilibrium under conditions of risk," The Journal of Finance, vol. 19, no. 3, pp. 425-442, 1964.

[4] M. J. Lynge and J. K. Zumwalt, "An empirical study of the interest rate sensitivity of commercial bank returns: a multiindex approach," Journal of Financial and Quantitative Analysis, vol. 15, no. 3, pp. 731-742, 1980.

[5] M. R. Morey and R. C. Morey, "Mutual fund performance appraisals: a multi-horizon perspective with endogenous benchmarking," Omega, vol. 27, no. 2, pp. 241-258, 1999.

[6] W. Briec, K. Kerstens, and J. B. Lesourd, "Single-period Markowitz portfolio selection, performance gauging, and duality: a variation on the Luenberger shortage function," Journal of Optimization Theory and Applications, vol. 120, no. 1, pp. 1-27, 2004.

[7] A. Kane, "Skewness preference and portfolio choice," The Journal of Financial and Quantitative Analysis, vol. 17, no. 1, pp. $15-25,1982$.

[8] F. D. Arditti, "Skewness and investors' decisions: a reply," The Journal of Financial and Quantitative Analysis, vol. 10, no. 1, pp. 173-176, 1975.

[9] Y. K. Ho and Y. L. Cheung, "Behavior of intra-daily stock return on an Asian emerging market-Hong Kong," Applied Economics, vol. 23, no. 5, pp. 957-966, 1991.

[10] T. Joro and P. Na, "Portfolio performance evaluation in a meanvariance-skewness framework," European Journal of Operational Research, vol. 175, no. 1, pp. 446-461, 2006.

[11] P. B. Hazell, "A linear alternative to quadratic and semivariance programming for farm planning under uncertainty," American Journal of Agricultural Economics, vol. 53, no. 1, pp. 53-62, 1971.

[12] J. Estrada, "Mean-semivariance behavior: downside risk and capital asset pricing," International Review of Economics \& Finance, vol. 16, no. 2, pp. 169-185, 2007.

[13] M. S. Bazaraa, H. D. Sherali, and C. M. Shetty, Nonlinear Programming: Theory and Algorithms, John Wiley \& Sons, New York, NY, USA, 2013.

[14] C. Buchanan, Techniques for solving nonlinear programming problems with emphasis on interior point methods and optimal control problems [Master of Philosophy], Department of Mathematics and Statistics, University of Edinburgh, 2008.

[15] K. E. Atkinson, An Introduction to Numerical Analysis, John Wiley \& Sons, New York, NY, USA, 2008.

[16] M. W. Davis, "Production of conditional simulations via the LU triangular decomposition of the covariance matrix," Mathematical Geology, vol. 19, no. 2, pp. 91-98, 1987.
[17] D. M. Hawkins and W. J. R. Eplett, "The Cholesky factorization of the inverse correlation or covariance matrix in multiple regression," Technometrics, vol. 24, no. 3, pp. 191-198, 1982.

[18] C. Hassold, The Valuation of Multivariate Options, diplom. De, 2004.

[19] D. Duffie and J. Pan, "An overview of value at risk," The Journal of Derivatives, vol. 4, no. 3, pp. 7-49, 1997.

[20] C. Genest and A.-C. Favre, "Everything you always wanted to know about copula modeling but were afraid to ask," Journal of Hydrologic Engineering, vol. 12, no. 4, pp. 347-368, 2007.

[21] B. Mandelbrot, "The variation of certain speculative prices," Journal of Business, vol. 36, no. 4, pp. 394-419, 1963.

[22] I. Popova, E. Popova, D. Morton, and J. Yau, "Optimal hedge fund allocation with asymmetric preferences and distributions," SSRN, 900012, 2006.

[23] R. J. Davies, H. M. Kat, and S. Lu, "Fund of hedge funds portfolio selection: a multiple-objective approach," Journal of Derivatives \& Hedge Funds, vol. 15, no. 2, pp. 91-115, 2009.

[24] L. T. DeCarlo, "On the meaning and use of kurtosis," Psychological Methods, vol. 2, no. 3, pp. 292-307, 1997.

[25] W. Briec, K. Kerstens, and O. Jokung, "Mean-variance-skewness portfolio performance gauging: a general shortage function and dual approach," Management Science, vol. 53, no. 1, pp. 135-149, 2007.

[26] A. S. Taylan and H. Tatlıdil, "Portfolio optimization with short function and higher order moments: an application in ISE-30," in Proceedings of the 24th Mini EURO Conference, Continuous Optimization and Information-Based Technologies in the Financial Sector (MEC EurOPT '10), İzmir, Turkey, June 2010.

[27] K. K. Lai, L. Yu, and S. Wang, "Mean-variance-skewnesskurtosis-based portfolio optimization," in Proceedings of the 1st International Multi-Symposiums on Computer and Computational Sciences (IMSCCS '06), vol. 2, pp. 292-297, Hanzhou, China, April 2006.

[28] B. Aracioglu, F. Demircan, and H. Soyuer, "Mean-varianceskewness-kurtosis approach to portfolio optimization: an application in Istanbul stock exchange, IMKB Uygulamasi," Ege Akademik Bakis, vol. 11, pp. 9-17, 2011. 


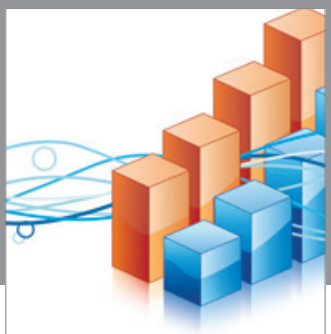

Advances in

Operations Research

vatem alat4

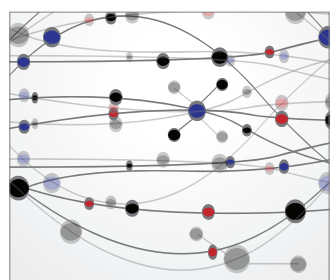

\section{The Scientific} World Journal
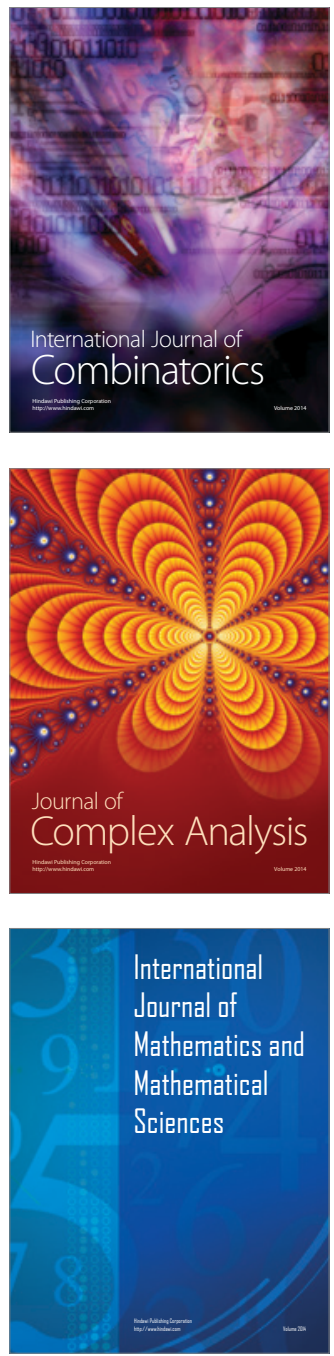
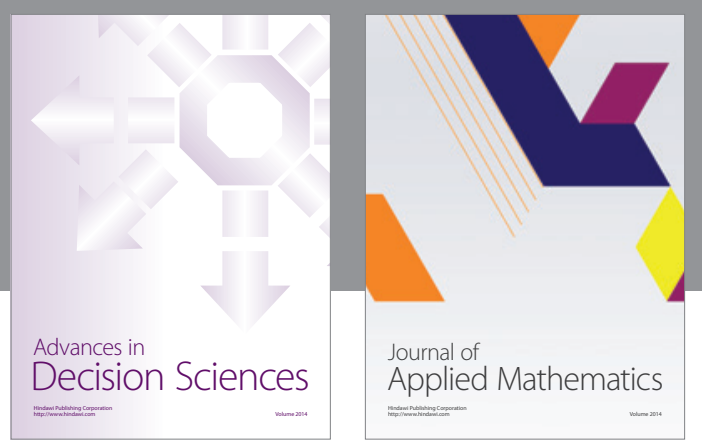

Algebra

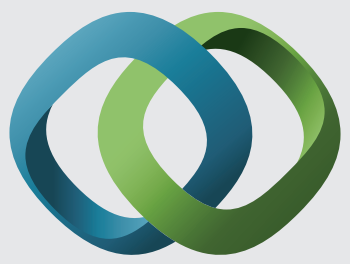

\section{Hindawi}

Submit your manuscripts at

http://www.hindawi.com
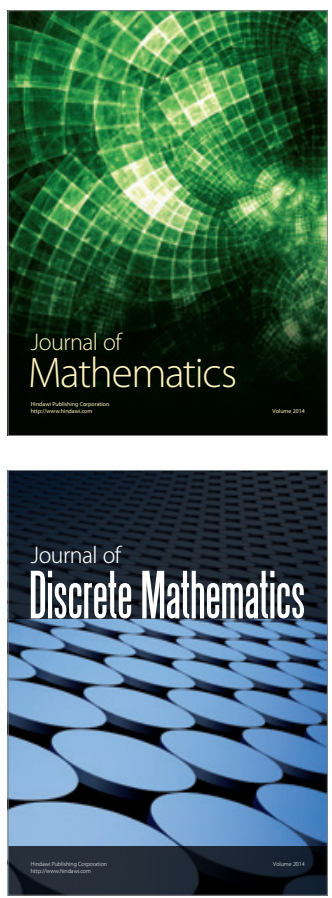

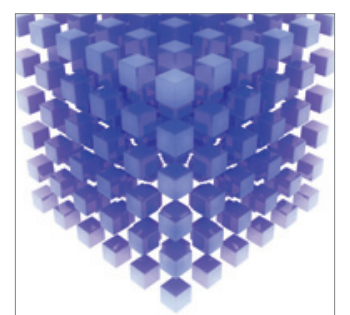

Mathematical Problems in Engineering
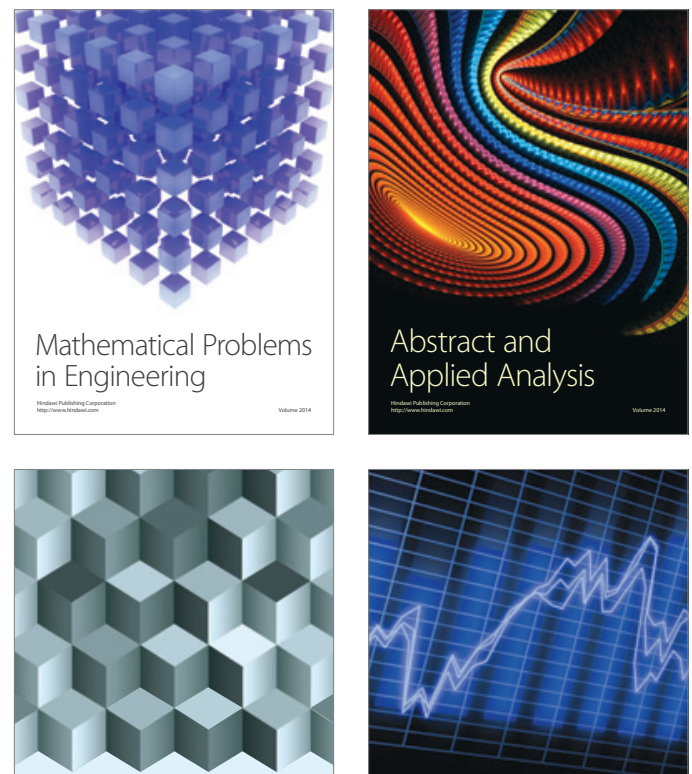

Journal of

Function Spaces

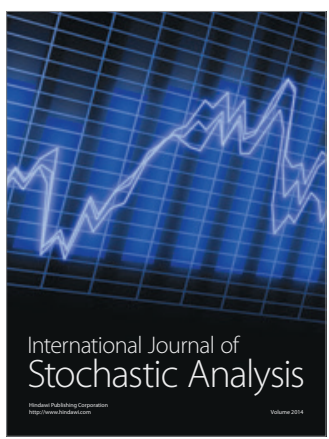

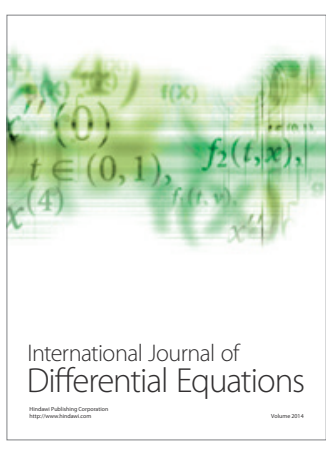
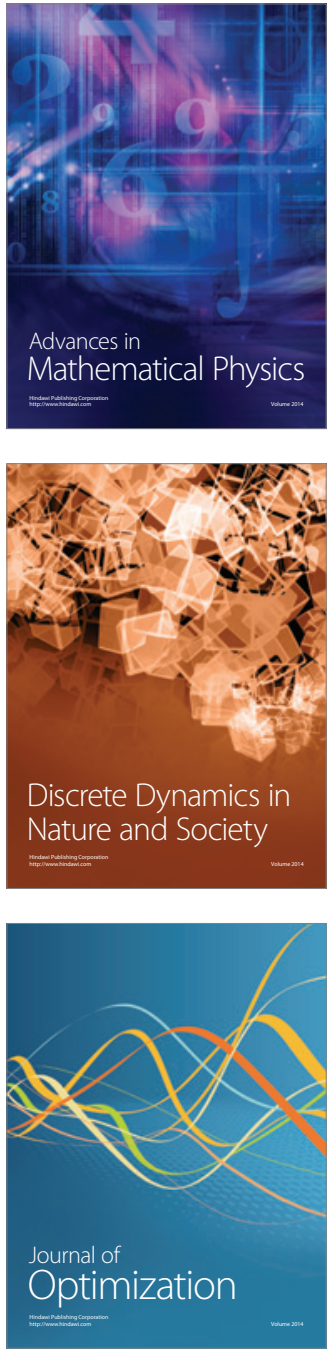